\title{
FUNDAMENTAL TWO-DIMENSIONAL SOLUTIONS OF ELECTROELASTICITY FOR COMPOUND PIEZOCERAMIC BODIES
}

\section{A. Fil'shtinskii}

UDC 539.3

We construct fundamental solutions of the two-dimensional equations of electroelasticity for antiplanar strain of a piezoceramic space with an interphase defect. We study the orders of the powers of the singularities at the vertices of the defect for two cases: an interphase crack and a stiff fiber continuously joined to the upper halfspace which has flaked off the lower half-space. The solution is constructed in closed form.

Bibliography: 4 titles.

Kosmodamianskii et al. [1] have constructed the Green's function for a piezoceramic half-plane with elliptic holes. In the present paper we use fundamental solutions of the corresponding equations of electroelasticity in constructing integral representations of the solutions of boundary-value problems.

We consider an antiplanar strain of a compound piezoceramic space under concentrated forces. Suppose the space is composed of two inhomogeneous piezoceramic half-spaces (polarized along the $x_{3}$-axis) continuously conjoined along the plane $x_{2}=0$ and is referred to Cartesian axes $O x_{1} x_{2} x_{3}$. There is a constant shear load $X_{3}=-p_{1}$ $\left(X_{1}=X_{2}=0\right)$ along the fiber $x_{1}=x_{10}, x_{2}=x_{20}>0,-\infty<x_{3}<\infty$ that is continuously distributed, or an electric charge of intensity $p_{2}$, and at infinity the components of the stress tensor $\sigma_{i 3}$ and the electric field intensity vector $E_{m}$ are zero. Under these conditions the compound space is in a state of antiplanar strain, all the field quantities being expressible in terms of four analytic functions $F_{v}^{(r)}(z)(v, r=1,2)$ as follows [2]:

$$
\begin{aligned}
& u_{3}^{(r)}=\operatorname{Re} f_{1}^{(r)}(z), \quad \varphi^{(r)}=\operatorname{Re} f_{2}^{(r)}(z), E_{1}^{(r)}-i E_{2}^{(r)}=-F_{2}^{(r)}(z) \\
& \sigma_{13}^{(r)}-i \sigma_{23}^{(r)}=\sum_{v=1}^{2} d_{1 v}^{(r)} F_{v}^{(r)}(z), D_{1}^{(r)}-i D_{2}^{(r)}=\sum_{v=1}^{2} d_{2 v}^{(r)} F_{v}^{(r)}(z) .
\end{aligned}
$$

Here

$$
d_{11}=c_{44}, \quad d_{12}=d_{21}=e_{15}, \quad d_{22}=-\varepsilon_{11}, \quad z=x_{1}+i x_{2}, \quad F_{\mathrm{v}}(z)=d f_{\mathrm{v}}(z) / d z
$$

$\varphi, u_{3}$ and $D_{m}$ are respectively the electric potential and components of the vectors of elastic displacement and electric induction; $c_{44}=c_{44}^{E}, e_{15}$ and $\varepsilon_{11}=\varepsilon_{11}^{S}$ are the shear modulus measured with zero electric field, the piezomodulus, and the dielectric permittivity measured at zero strain [3]; the subscripts 1 and 2 refer to quantities defined in the upper and lower half-spaces.

The solution of this problem (the fundamental solution for a compound space) has the form [2]

$$
\begin{gathered}
\Phi_{v}^{(1)}(z)=\frac{A_{v}^{(1)}}{z-z_{0}}+\sum_{m=1}^{2} \frac{\alpha_{v, m}^{(1)} \bar{A}_{m}^{(1)}}{z-\bar{z}_{0}}, \operatorname{Im} z \geq 0(v=1,2) ; \\
\Phi_{v}^{(2)}(z)=\sum_{m=1}^{2} \frac{\alpha_{v+2, m}^{(1)} A_{m}^{(1)}}{z-z_{0}}, \operatorname{Im} z \leq 0, z_{0}=x_{10}+i x_{20},
\end{gathered}
$$

where

$$
\begin{aligned}
& A_{1}^{(1)}=\frac{p_{1} \varepsilon_{11}^{(1)}+p_{2} e_{15}^{(1)}}{2 \pi c_{44}^{(1)} \varepsilon_{11}^{(1)}\left(1+k_{1}^{2}\right)}, A_{2}^{(1)}=\frac{p_{1} e_{15}^{(1)}-p_{2} c_{44}^{(1)}}{2 \pi c_{44}^{(1)} \varepsilon_{11}^{(1)}\left(1+k_{1}^{2}\right)}, k_{1}^{2}=\frac{\left(e_{15}^{(1)}\right)^{2}}{c_{44}^{(1)} \varepsilon_{11}^{(1)}}, \\
& \Delta_{1} \alpha_{1,1}^{(1)}=\left(1-c_{1}\right)\left(1+\varepsilon_{1}\right)+k_{1}^{2}\left(1-e_{1}^{2}\right), \Delta_{1} \alpha_{2.1}^{(1)}=2 e_{15}^{(1)}\left(e_{1}-c_{1}\right) / \varepsilon_{11}^{(1)},
\end{aligned}
$$

Translated from Teoreticheskaya i Prikladnaya Mekhanika, No. 28, 1998, pp. 62-67. Original article submitted February 10, 1998. 


$$
\begin{aligned}
& \Delta_{1} \alpha_{1.2}^{(1)}=2 e_{15}^{(1)}\left(\varepsilon_{1}-e_{1}\right) / c_{44}^{(1)}, \Delta_{1} \alpha_{2,2}^{(1)}=\left(1+c_{1}\right)\left(1-\varepsilon_{1}\right)+k_{1}^{2}\left(1-e_{1}^{2}\right), \\
& \Delta_{1}=\left(1+c_{1}\right)\left(1+\varepsilon_{1}\right)+k_{1}^{2}\left(1+e_{1}\right)^{2}, \quad c_{1}=\frac{c_{44}^{(2)}}{c_{44}^{(1)}}, e_{1}=\frac{e_{15}^{(2)}}{e_{15}^{(1)}}, \varepsilon_{1}=\frac{\varepsilon_{11}^{(2)}}{\varepsilon_{11}^{(1)}} .
\end{aligned}
$$

In the case of antiplanar strain the complex representations of the stresses and displacements have the form

$$
u_{3}^{(r)}=\operatorname{Re} f^{(r)}\left(z_{r}\right), \quad \sigma_{13}^{(r)}=\sqrt{\Delta_{r}} \operatorname{Im}\left\{\mu^{(r)} F^{(r)}\left(z_{r}\right)\right\}, \quad \sigma_{23}^{(r)}=-\sqrt{\Delta_{r}} \operatorname{Im} F^{(r)}\left(z_{r}\right) .
$$

Here

$$
\begin{gathered}
\Delta_{r}=c_{44}^{(r)} c_{55}^{(r)}-\left(c_{45}^{(r)}\right)^{2}>0 ; \quad z_{r}=x_{1}+\mu^{(r)} x_{2} ; \\
\mu^{(r)}=\left(i \sqrt{\Delta_{r}}-c_{45}^{(r)}\right) / c_{44}^{(r)}(r=1,2) ; \quad F(x)=d f(x) / d x ;
\end{gathered}
$$

$c_{i j}$ are the corresponding parameters of elasticity of the anisotropic medium [4].

The required analytic functions can be determined from the coupling conditions for the half-spaces along the plane $x_{2}=0$ and the static conditions in a neighborhood of the point $z_{10}=x_{10}+i x_{20}$. We have

$$
\begin{gathered}
F^{(1)}\left(z_{1}\right)=A\left(\frac{1}{z_{1}-z_{10}}+\frac{\alpha}{z_{1}-\bar{z}_{10}}\right), \quad \operatorname{Im} z_{1} \geq 0 ; F^{(2)}\left(z_{2}\right)=\frac{A \beta}{z_{2}-z_{10}}, \operatorname{Im} z_{2} \leq 0 ; \\
\alpha=\frac{1-\gamma}{1+\gamma}, \quad \beta=\frac{2}{1+\gamma}, \quad \gamma=\sqrt{\frac{\Delta_{2}}{\Delta_{1}}} .
\end{gathered}
$$

Consider a piezoceramic space with an interphase band crack. Suppose that flaking occurs along the band $-a<x_{1}<b, x_{2}=0,-\infty<x_{3}<\infty$, on the interface of the materials, which we shall treat as an interphase crack. We take the load as in (1)-(2). We shall assume that the surface of the crack is free of forces, while the electric boundary conditions have the following form [3]: $E_{1}^{(1)}=E_{1}^{(2)}, D_{1}^{(1)}=D_{1}^{(2)}$ when $x_{2}=0,-a<x_{1}<b$.

Then the solution, which we shall call the Green's function, is determined by the following relations [2]:

$$
\begin{gathered}
F_{\mathrm{v}}^{(1)}(z)=\Phi_{\mathrm{v}}^{(1)}(z)-2 \omega_{\mathrm{v}}^{(1)} \Phi(z), \quad \operatorname{Im} z \geq 0(v=1,2), \\
F_{\mathrm{v}}^{(2)}(z)=\Phi_{\mathrm{v}}^{(2)}(z)-2 \omega_{\mathrm{v}}^{(2)} \Phi(z), \operatorname{Im} z \leq 0, \\
\Phi(z)=\frac{C}{4 \pi}\left\{\frac{1-X\left(\bar{z}_{0}\right) X^{-1}(z)}{z-\bar{z}_{0}}-\frac{1-X\left(z_{0}\right) X^{-1}(z)}{z-z_{0}}\right\}, \\
C=\Delta_{2} \sum_{m=1}^{2} p_{m} B_{m}, \quad B_{m}=-\frac{\omega_{m}^{(1)}}{B c_{44}^{(1)}}, \quad B=\frac{1}{\varepsilon_{1}}\left(1+\varepsilon_{1}+k_{1}^{2}\left(1+\frac{e_{1}^{2}}{c_{1}}\right)\right), \\
\omega_{1}^{(1)}=\frac{1}{2}\left(1-\alpha_{1.1}^{(1)}\right), \omega_{2}^{(1)}=-\frac{1}{2} \alpha_{2,1}^{(1)}, \omega_{1}^{(2)}=\frac{1}{2}\left(1+\alpha_{1,1}^{(1)}\right), \omega_{2}^{(2)}=-\omega_{2}^{(1)}, \\
X(z)=\sqrt{(z+a)(z-b)}, \quad \Delta_{2}=\Delta_{1} /\left(c_{1} \varepsilon_{1}\right) .
\end{gathered}
$$

The other constants and the functions $\Phi_{v}^{(r)}(z)$ are defined in (2).

The stress intensity factors and the electric field intensity at the vertices of the interphase crack can be computed from the formulas

$$
\begin{gathered}
K_{I I I}^{+}=\frac{\Lambda}{\sqrt{2 L}} \operatorname{Im} k^{-1}\left(z_{0}\right), K_{I I I}^{-}=-\frac{\Lambda}{\sqrt{2 L}} \operatorname{Im} k\left(z_{0}\right), \quad K_{E}^{ \pm}=\frac{e_{15}^{(1)}\left(e_{1}-c_{1}\right)}{B c_{44}^{(2)} \varepsilon_{11}^{(2)}} K_{I I I}^{ \pm} ; \\
\Lambda=\frac{1}{\pi} \sum_{m=1}^{2} p_{m} \omega_{m}^{(1)}, \quad k(x)=\sqrt{\frac{x-b}{x+a}}, \quad 2 L=a+b .
\end{gathered}
$$

Now let us consider a compound piezoceramic space, and suppose that on the interface between the materials $x_{2}=0$ there is a thin stiff inclusion occupying the band $x_{1} \in(-a, b)=l,-\infty<x_{3}<\infty$. We shall assume that the band is continuously connected to the upper half-space and disconnected (at all points) from the lower half-space. The loading of the compound body is done just as in (1)-(2). We assume the boundary conditions on $l$ in the form 


$$
\partial_{1} u_{3}^{(1)}=0, \quad \sigma_{23}^{(2)}=0, E_{1}^{(1)}=E_{1}^{(2)}, D_{2}^{(1)}=D_{2}^{(2)} .
$$

For $x_{1} \bar{\epsilon} /$ we have the usual coupling conditions for two media. The solution of this boundary-value problem is determined by the formulas

$$
\begin{gathered}
F_{v}^{(1)}(z)=\Phi_{v}^{(1)}(z)+i \sum_{m=1}^{2} a_{1 m}^{(v)} \Phi_{m}(z), \quad \operatorname{Im} z \geq 0 \quad(v=1,2), \\
F_{v}^{(2)}(z)=\Phi_{v}^{(2)}(z)+i \sum_{m=1}^{2} a_{2 m}^{(v)} \Phi_{m}(z), \quad \operatorname{Im} z \leq 0, \\
i \Phi_{1}(z)=\frac{\Delta}{4 \pi} X_{01}(z) \sum_{v=1}^{2} p_{v}\left\{a_{11}^{(v)} \Pi_{1}\left(z, z_{0}\right)+a_{11}^{(v)} \Pi_{1}\left(z, \bar{z}_{0}\right)\right\}, \\
i \Phi_{2}(z)=-\frac{\Delta}{4 \pi} X_{02}(z) \sum_{v=1}^{2} p_{v}\left\{a_{11}^{(v)} \Pi_{2}\left(z, z_{0}\right)+\bar{a}_{11}^{(v)} \Pi_{2}\left(z, \bar{z}_{0}\right)\right\}, \\
\Pi_{m}\left(z, z_{0}\right)=1-\frac{X_{m}(z)-X_{m}\left(z_{0}\right)}{z-z_{0}}, \Delta=\frac{\Delta_{2}}{B c_{44}^{(1)}}, \\
X_{m}(z)=X_{0 m}^{-1}(z)=(z+a)^{\gamma_{m}}(z-b)^{1-\gamma_{m}} .
\end{gathered}
$$

The exponents $\gamma_{m}$ that occur in the canonical solution $X_{0 m}(z)$ can be computed using the relations

$$
\gamma_{m}=\frac{\theta_{m}}{2 \pi}, \cos \theta_{m}=\frac{1-\gamma^{2}}{1+\gamma^{2}}, \sin \theta_{m}=(-1)^{m-1} \frac{2 \gamma}{1+\gamma^{2}}(m=1,2), \quad \gamma=\lambda B c_{1} \varepsilon_{1}\left(\omega_{1}^{(1)} \Delta_{1}\right)^{-1}, \lambda^{2}=\frac{1+\varepsilon_{1}}{c_{1} \varepsilon_{1} B} \text {. }
$$

Thus the exponent of a power singularity in the mechanical stresses or electric field intensity and induction at the ends of the defect depends strongly on the physical parameters of the materials of the composite. equality

The contact stress on the line of coupling between the upper half-space and the stiff fiber is defined by the

$$
\begin{gathered}
\sigma_{23}^{(1)}(x)=\frac{c_{44}^{(1)}}{2 \lambda}\left(W_{2}(x)-W_{1}(x)\right), x \in l, \\
W_{m}(x)=-\frac{\gamma \Delta U_{m}(x)}{\pi \sqrt{1+\gamma^{2}}} \operatorname{Re} \sum_{v=1}^{2} p_{v} a_{m v}\left(1+\frac{X_{m}\left(z_{0}\right)}{x-z_{0}}\right)(m=1,2), \\
a_{1 v}=\bar{a}_{11}^{(v)}, a_{2 v}=a_{11}^{(v)}, \\
U_{m}(x)=(x+a)^{-\theta_{m} / 2 \pi}(b-x)^{\theta_{m} / 2 \pi-1}, \operatorname{Im} U_{m}(x)=0, x \in l .
\end{gathered}
$$

Consequently $\sigma_{23}^{(1)}(x)$ also has a power singularity at the ends of the interval $l$.$$
\text { Literature Cited }
$$

1. A. S. Kosmodamianskii, A. P. Kravchenko, and V. N. Lozhkin, "The action of a point electric charge on the boundary of a piezoelectric half-plane weakened by an elliptic hole," lzv. Akad. Nauk Arm. SSR. Mekh., 30, No. 1, 13-20 (1977).

2. L. A. Fil'shtinskii and M. L. Fil'shtinskii, "Antiplanar strain of a compound piezeceramic space with an interphase crack," Prikl. Mekh., 33, No. 8, 73-78 (1997).

3. V. T. Grinchenko, A. F. Ulitko, and N. A. Shul'ga, Mechanics of Coupled Fields in Structural Elements in Russian], Naukova Dumka, Kiev (1989).

4. A. S. Kosmodamianskii, The Stressed State of Anisotropic Media with Holes or Cavities [in Russian], Vishcha Shkola, Kiev (1976). 